\title{
SOLAR ENERGETIC PARTICLE VARIATIONS
}

\author{
D. V. Reames \\ NASA Goddard Space Flight Center, Greenbelt, MD 20771, USA
}

\begin{abstract}
In the largest solar energetic-particle (SEP) events, acceleration occurs at shock waves driven out from the Sun by coronal mass ejections (CMEs). In fact, the highest proton intensities directly measured near Earth at energies up to $\sim 1 \mathrm{GeV}$ occur at the time of passage of shocks, which arrive about a day after the CMEs leave the Sun. CMEdriven shocks expanding across magnetic fields can fill over half of the heliosphere with SEPs. Proton-generated Alfven waves trap particles near the shock for efficient acceleration but also throttle the intensities at Earth to the "streaming limit" early in the events. At high energies, particles begin to leak from the shock and the spectrum rolls downward to form an energy-spectral "knee" that can vary in energy from $\sim 1 \mathrm{MeV}$ to $\sim 1 \mathrm{GeV}$ in different events. All of these factors affect the radiation dose as a function of depth and latitude in the Earth's atmosphere and the risk to astronauts and equipment in space. SEP ionization of the polar atmosphere produces nitrates that precipitate to become trapped in the polar ice. Observations of nitrate deposits in ice cores reveal individual large SEP events and extend back $\sim 400$ years. Unlike sunspots, SEP events follow the $\sim 80-100$-year Gleissberg cycle rather faithfully and are now at a minimum in that cycle. The largest SEP event in the last 400 years appears to be related to the flare observed by Carrington in 1859 , but the probability of SEP events with such large fluences falls off sharply because of the streaming limit.
\end{abstract}

\section{INTRODUCTION}

The solar wind, which blows at speeds of 300 to $800 \mathrm{~km} / \mathrm{s}$, corresponds to proton energies of 0.5 to $3 \mathrm{keV}$. The protons in solar energetic-particle (SEP) events have energy spectra that span the region from about $10 \mathrm{keV}$ to $>10 \mathrm{GeV}$. In large SEP events, omni-directional fluences of $>30 \mathrm{MeV}$ protons can exceed $10^{10} \mathrm{~cm}^{-2}$; protons of this energy can penetrate spacecraft walls and astronaut's space suits, and plunge deeply into the polar atmosphere. The events can have rapid onsets and durations from several hours to several days. While protons are the dominant particle species in SEP events, there are also ions of elements throughout the periodic table. Abundances and ionization states of these ions have made a major contribution to our understanding of the particle acceleration and transport and the nature of the source plasma.

We have identified two distinct classes of SEP events, called "impulsive" and "gradual" (or long-duration), which correspond to two physical mechanisms of particle acceleration (Reames 1990, 1995, 1999, 2002; Kahler 1992, 2001; Gosling 1993; Tylka 2001). Impulsive SEP events have unusual 1000-fold enhancements, relative to coronal abundances, of ${ }^{3} \mathrm{He} /{ }^{4} \mathrm{He}$ and heavy elements $(\mathrm{Z} \geq 50) / \mathrm{O}$ that are believed to result from resonant waveparticle interactions turbulent regions of magnetic reconnection in solar flares. Impulsive SEP events come from a small interval of solar longitude that is magnetically well connected to the observer and the ions are highly ionized by the hot $(>10 \mathrm{MK})$ flare plasma. In contrast, the particles in gradual SEP events are accelerated at shock waves driven out from the Sun by fast coronal mass ejections (CMEs) so that high particle intensities can be seen for days spanning more than $200^{\circ}$ in solar longitude. Element abundances and ionization states in gradual events are similar to those of the ambient (1-2 MK) plasma of the corona or solar wind, although interesting departures do occur (Reames 1999; Tylka et al. 1999; Reames et al. 2000; Tylka 2001). Since all of the largest events of any practical significance are gradual events, we will not discuss impulsive events in the remainder of this paper.

Until recently, our view of the "climate" of SEP events was limited to the observations of the last 40 years in space and ground-level observations in the two decades prior to space exploration. However, recent high-resolution 
observations of nitrate deposits in polar ice cores (McCracken et al. 2001a, b) have begun to give us a synoptic look at properties of large SEP events that have occurred during the last $\sim 400$ years. These observations address the probabilities that very large SEP events occur and the long-time-scale cycles of the SEP climate.

In this paper, we first examine the physics of SEP acceleration and transport and the resulting properties of the events and their variations in space, time, and particle energy. We then discuss event cycles and the size distribution of events and the prospects for event prediction.

\section{SHOCK ACCELERATION AND SHOCK PEAKS}

The highest intensities of $<1 \mathrm{GeV}$ protons we have seen near Earth occurred at intensity peaks near times of shock passage. The SEP event of 1972 August 4 is one of the largest events seen since measurements in space have been available. Figure 1 compares fluxes in this event with those in recent events. Unfortunately, the energy intervals of the Explorer 41 instrument did not correspond well with those on the GOES spacecraft in the later events. However, peak fluxes of $>60 \mathrm{MeV}$ protons near the shock on 1972 August 4 even exceed the peaks of the $>40 \mathrm{MeV}$ protons in the later events.

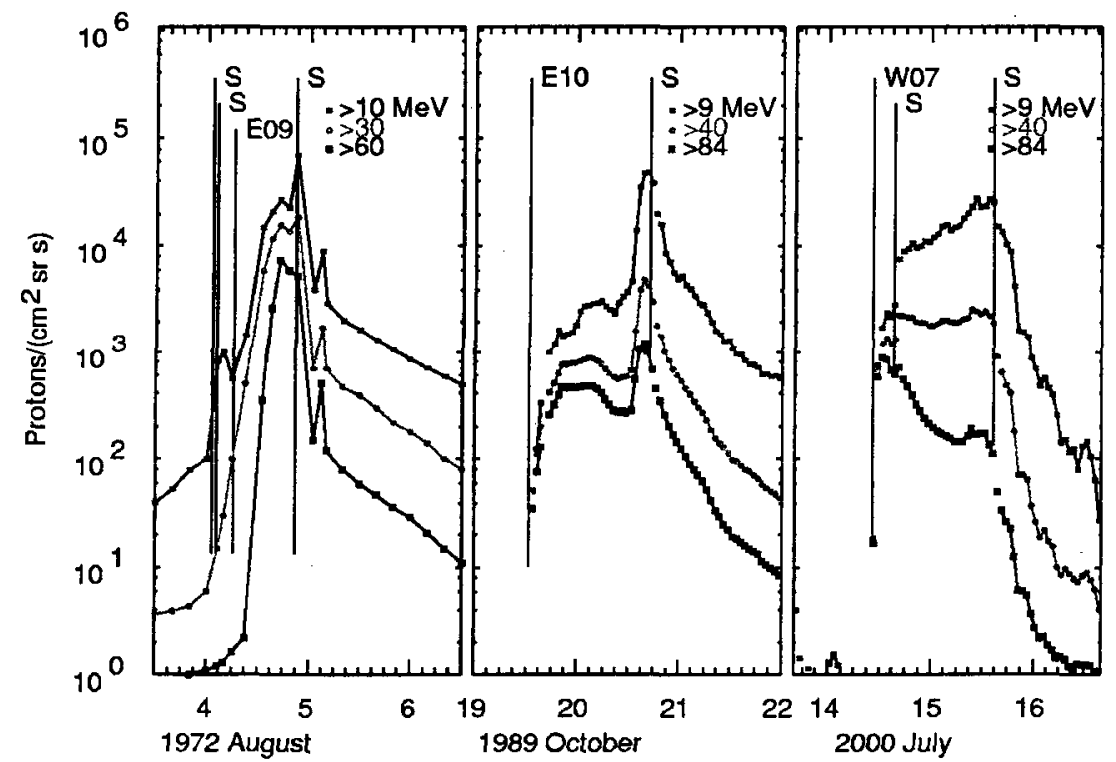

Fig 1. The flux of protons often peaks at the time of shock passage, as seen in three large SEP events

Particles gain energy at shock waves when they resonantly scatter back and forth against Alfvén waves upstream and downstream of the shock, gaining twice the shock velocity on each round trip (see Jones and Ellison 1991). As the particles stream away from the shock, they amplify Alfvén waves with wave vector $k=B / \mu P$, where $B$ is the magnetic field strength, $P$ is the particle rigidity or momentum per unit charge, and $\mu$ is the cosine of the particle pitch angle with respect to the magnetic field. As the wave intensities increase, subsequent particles are more efficiently scattered and therefore trapped near the shock, thus they gain more energy and then amplify waves resonant with this higher energy. Thus, the acceleration proceeds to higher and higher energies. An equilibrium theory of acceleration by self-generated waves was developed for galactic cosmic rays by Bell (1978) and was applied to solar and interplanetary shocks by Lee $(1983,1997)$.

Nearly all of the waves are generated by protons, the most abundant particle species, while the other species, acting as test particles, probe the spectrum of the proton-generated waves. Ions other than $\mathrm{H}$ have differing chargeto-mass ratios $Q / A$, hence they probe different regions of the wave spectrum at a given velocity. For this reason, abundance ratios of different ion species, such as $\mathrm{He} / \mathrm{H}$ or $\mathrm{Fe} / \mathrm{O}$ explore the proton-generated wave spectrum and its variation with space and time, providing a powerful test of models of SEP events (e.g. Tylka et al. 1999; $\mathrm{Ng}$ et al. 1999; Reames et al. 2000; Tylka 2001). Particle angular distributions, which map SEP flows, also probe wave spectra and measure particle trapping (see Reames et al. 2001; Reames and $\mathrm{Ng} \mathrm{2002).} \mathrm{Abundances,} \mathrm{angular}$ distributions, and ionization states are all powerful tools that probe the physics of shock acceleration, but we will not have space to discuss these further in this paper. 
As shocks move outward from the Sun they weaken and the SEP energy spectra become steeper. However, Figure 2 shows that protons with energies up to $510-700 \mathrm{MeV}$ can still peak at a shock that is driven by a sufficiently fast $\mathrm{CME}$ coming from the region near central meridian on the Sun, such as the event of 2001 November 4. The event of 1989 October 19, shown at lower energies in Figure 1, also has a strong shock peak above $500 \mathrm{MeV}$.

Kahler et al. (1984) found that the speed of a CME is one of the most important parameters in determining the peak proton intensity, wherever the peak occurred during an event. Figure 3 shows a recent study of this correlation. Because of this correlation, only the fastest $\sim 1 \%$ of CMEs produce significant SEP events. Most CMEs are emitted nearly at the solar wind speed, thus they lack SEPs.

Kahler (2001) realized that spectral variation affects the correlation with CME speed. Even with a perfect correlation at $2 \mathrm{MeV}$, differences in spectral slope would cause a large spread at $20 \mathrm{MeV}$. The longitude and angular spread of a CME also affect the probability that the shock intercepts the observer's magnetic flux tube.

Of course, CME observations were not available for the 1972 August 4 event shown in Figure 1. However, the average speed of the shock, from the Sun to the Earth, in this event was $2900 \mathrm{~km} / \mathrm{s}$. This is well above the speed scale in Figure 3.

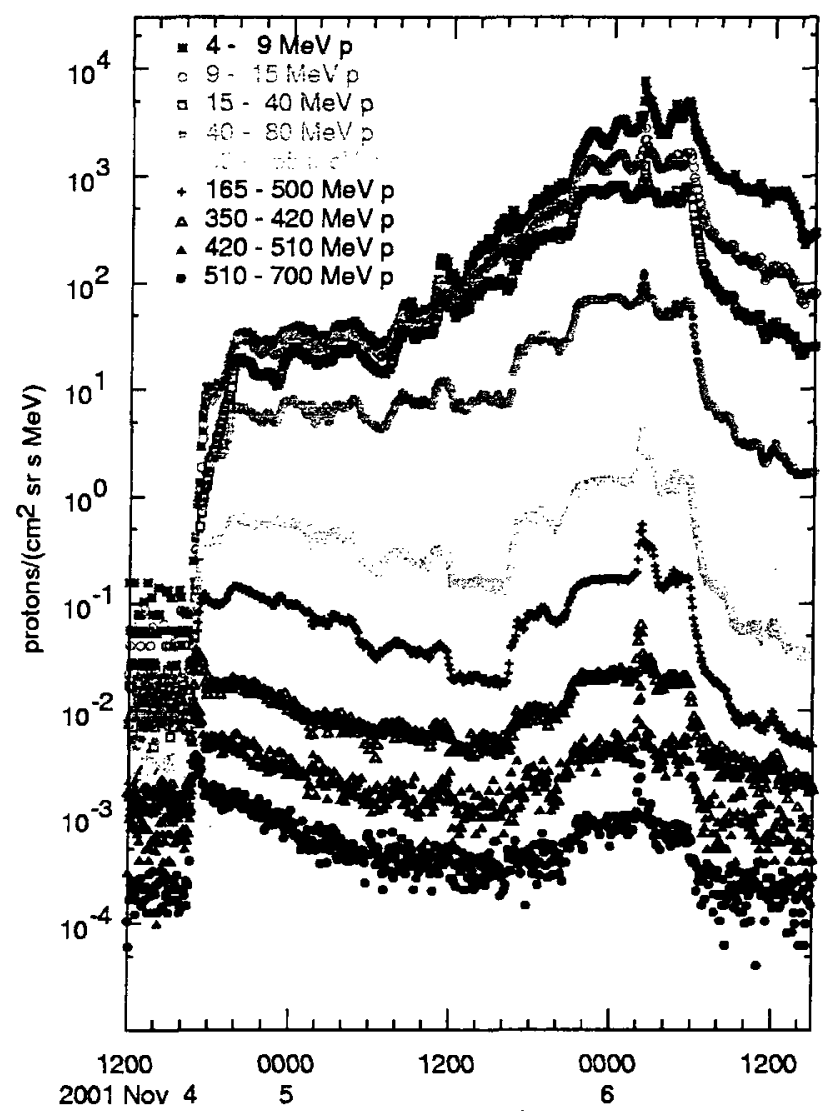

Fig 2. The peak at the time of shock passage is clearly defined early on November 6 , even at proton energies as high as $510-700 \mathrm{MeV}$.

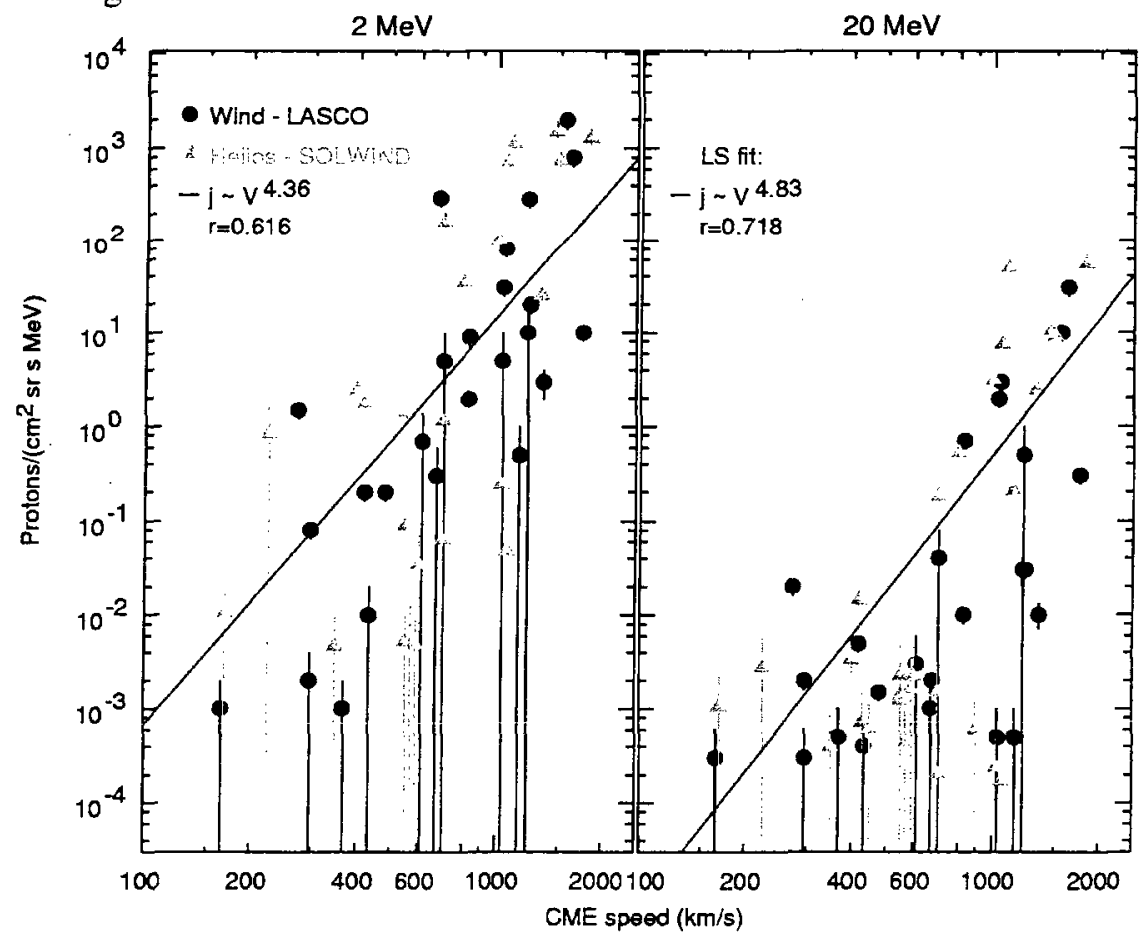

Fig 3. Least-squares fits of peak proton intensities vs. CME speeds are shown for two particle energies for events observed with two combinations of spacecraft (Kahler 2001). Fitted intensities, j, exceed the $4^{\text {th }}$ power of the CME speed and correlation coefficients, $r$, are high. 


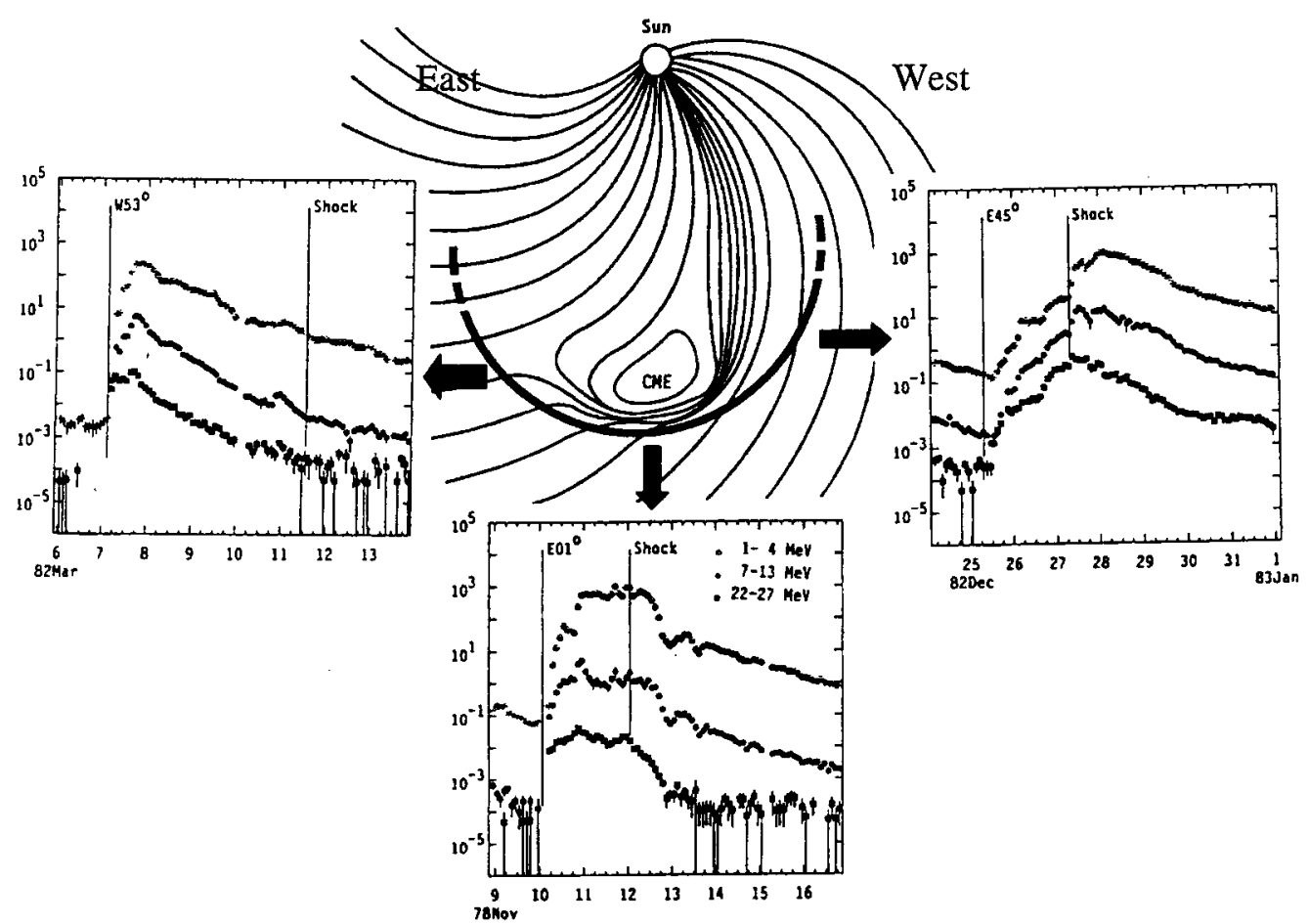

Fig 4. Intensity-time profiles for protons are shown for observers viewing a CME from three different longitudes (Cane et al. 1988; Reames 1999).

\section{PARTICLE DISTRIBUTIONS IN SPACE AND TIME}

Energetic particles are strongly constrained to follow magnetic field lines from their source to the observer. The Parker spiral pattern of the interplanetary field, produced by the solar rotation, causes an asymmetry in the intensity-time profiles depending upon the observer's longitude (and latitude) relative to the "nose" of the shock centered on the CME driver as shown in Figure 4. SEP intensities decrease as one moves along the shock front from the nose, where the shock speed is highest, around to the flanks on either side.

An observer on the east flank of the shock, as shown in Figure 4, sees a source at a western longitude (e.g. $\mathrm{W} 53^{\circ}$ ) on the Sun. As a function of time, this observer's connection point swings from the intense nose of the shock, near the Sun, to the weaker flank at $1 \mathrm{AU}$. In contrast, an observer on the west flank of the shock $(e . g$. E45 source in Figure 4) may see maximum intensity only after crossing through the shock into the region where field lines connect to the shock nose from behind.

A sufficiently fast $\mathrm{CME}$ near central meridian will produce an intensity peak at the time of shock passage (seen in Figures 1 and 2, but not in Figure 4), followed by a sharp decrease in intensity when the observer enters the CME or magnetic cloud, as seen in Figure 4. The reduced intensity inside the CME shows that little or no acceleration persists at the reconnection region behind the CME. Occasionally, however, new events at the Sun do inject a new population of energetic particles into this region in and behind the CME (Kahler and Reames 1991)

The typical intensity-time profiles shown in Figure 4 were derived from a study of 235 SEP events as a function of source longitude using IMP-8 observations (Cane et al. 1988). However, on some occasions it is possible to observe a single CME from spacecraft at multiple positions around it. Figures 5 and 6 show intensity-time profiles for 3-6 MeV protons and energy spectra in SEP events observed by Helios-1, Helios-2, and IMP-8 (Reames et al. 1996, 1997). The longitudes of the observing spacecraft are seen in the inset in the upper panel of each Figure; the CME is directed downward in these insets as it was in Figure 4.

In Figure 5, Helios 1 observes a source near central meridian so the proton intensity rises to a plateau value (near the streaming limit, described below), then peaks at the time of shock passage, and subsequently declines. Helios 2 and IMP 8, on the west flank of the shock, see an event that rises less and less rapidly. The strong decline in intensity with longitude suggests a CME with a relatively narrow longitude span. However, intensities at all three spacecraft eventually converge to the same intensity that declines slowly with time. This is the "invariant spectral region," where all intensities and spectra are the same over a large spatial region. In this region, particles are essentially trapped in a magnetic "bottle" defined by the CME, which slowly expands with time. Adiabatic deceleration in this expanding bottle preserves the spectral shape (Reames et al. 1996, 1997). 


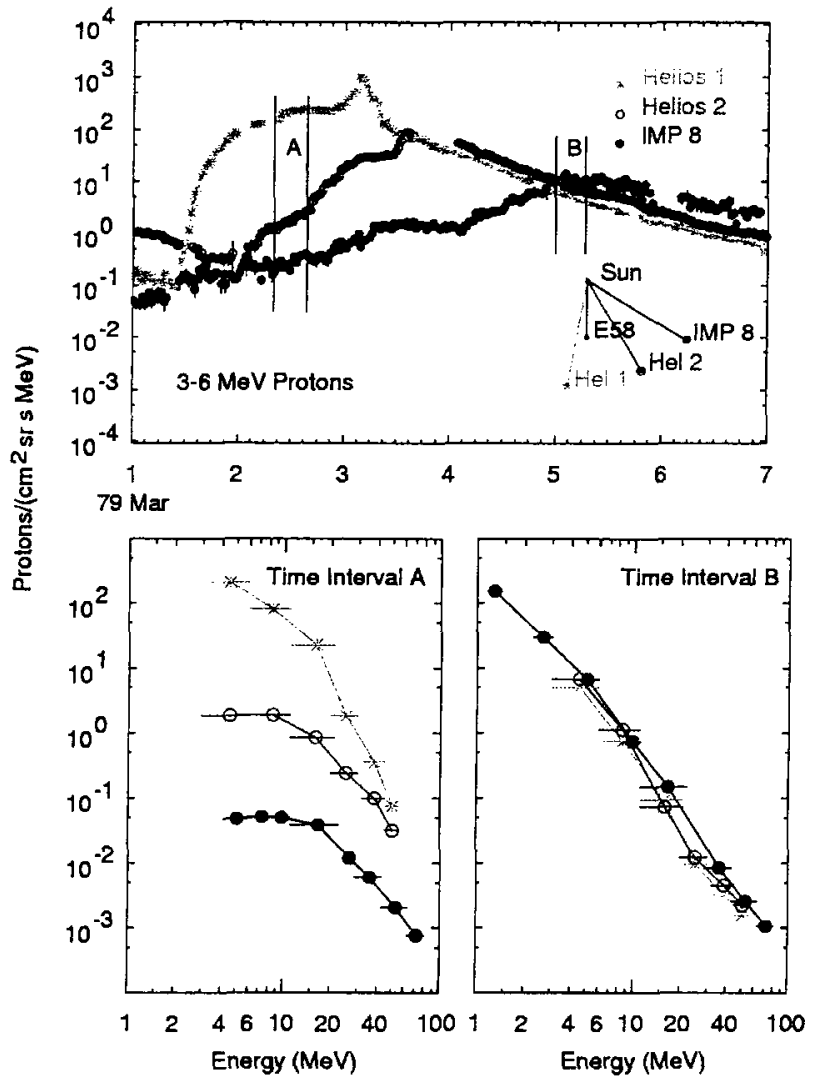

Fig. 5. Proton intensities and spectra observed by three spacecraft at different solar longitudes in the 1979 March 1 event (Reames et al. 1997).

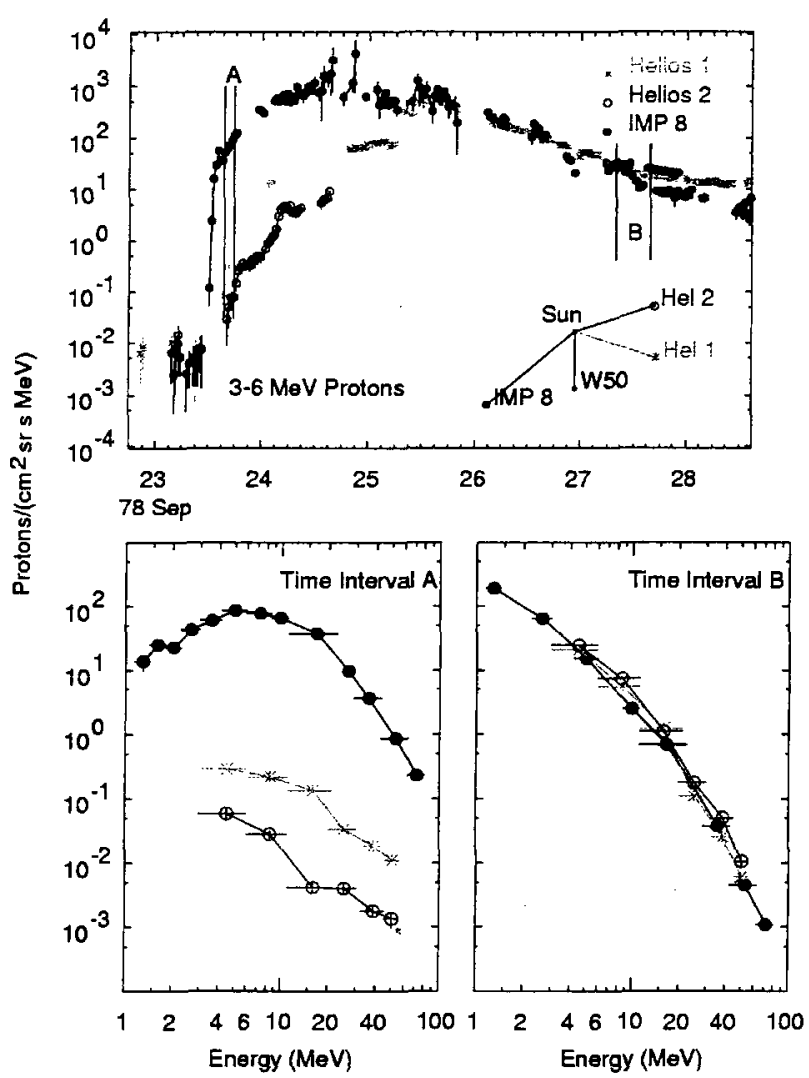

Fig. 6. Proton intensities and spectra observed by three spacecraft in the 1978 September 23 event (Reames et al. 1997).

The event in Figure 6 is much larger and more intense at all three spacecraft, yet they span a much larger angle of nearly $160^{\circ}$. The intensity at Helios 2 rises quite rapidly considering that the source is behind the east limb when viewed from Helios 2. The shock from this CME is seen at all three spacecraft. An invariant spectral region is again seen late in the event, despite the huge longitude span of the spacecraft.

In addition to the longitude dependence, the acceleration at a given point on the shock can decrease strongly because the shock weakens as it expands radially. This dependence can be especially strong at high energies. Kahler (1994) found that the intensities of $21 \mathrm{GeV}$ protons peaked when the shock was at 5-10 solar radii. Only the strongest shocks near central meridian produce shock peaks above $100 \mathrm{MeV}$ at $1 \mathrm{AU}$, as we have seen.

\section{THE STREAMING LIMIT}

Since we know that CME-driven shock waves are strongest when they are a few solar radii from the Sun (Kahler 1994) and they weaken as they come out toward Earth, it is surprising that the highest particle intensities occur late in the events when the shock itself arrives at Earth (see Figures 1 and 2). This occurs because particle intensities early in a large SEP event are bounded at the "streaming limit," while those near the shock are not. The streaming limit controls the transport of particles from their source to the observer.

As protons of a given rigidity stream away from a shock, they generate Alfvén waves which scatter protons coming behind, as described above. Near the shock, the proton and wave intensities reach an equilibrium when the proton steaming is just adequate to grow enough waves to compensate for the waves convected downstream (Lee 1983). The intensities of protons and resonant waves decrease as we move upstream of the shock along an expanding flux tube until we reach a point where the proton intensity is inadequate to produce sufficient wave intensity; from here the particles begin to stream freely away. If we were to increase the proton intensity near the shock, resonant waves would quickly grow to retard the increased streaming and steepen the local spatial gradient, but the intensities near Earth would change little.(see Ng and Reames 1994). Figure 7 shows intensities at three different energies that are bounded at the streaming limit early in several large SEP events (Reames and Ng 1998). 


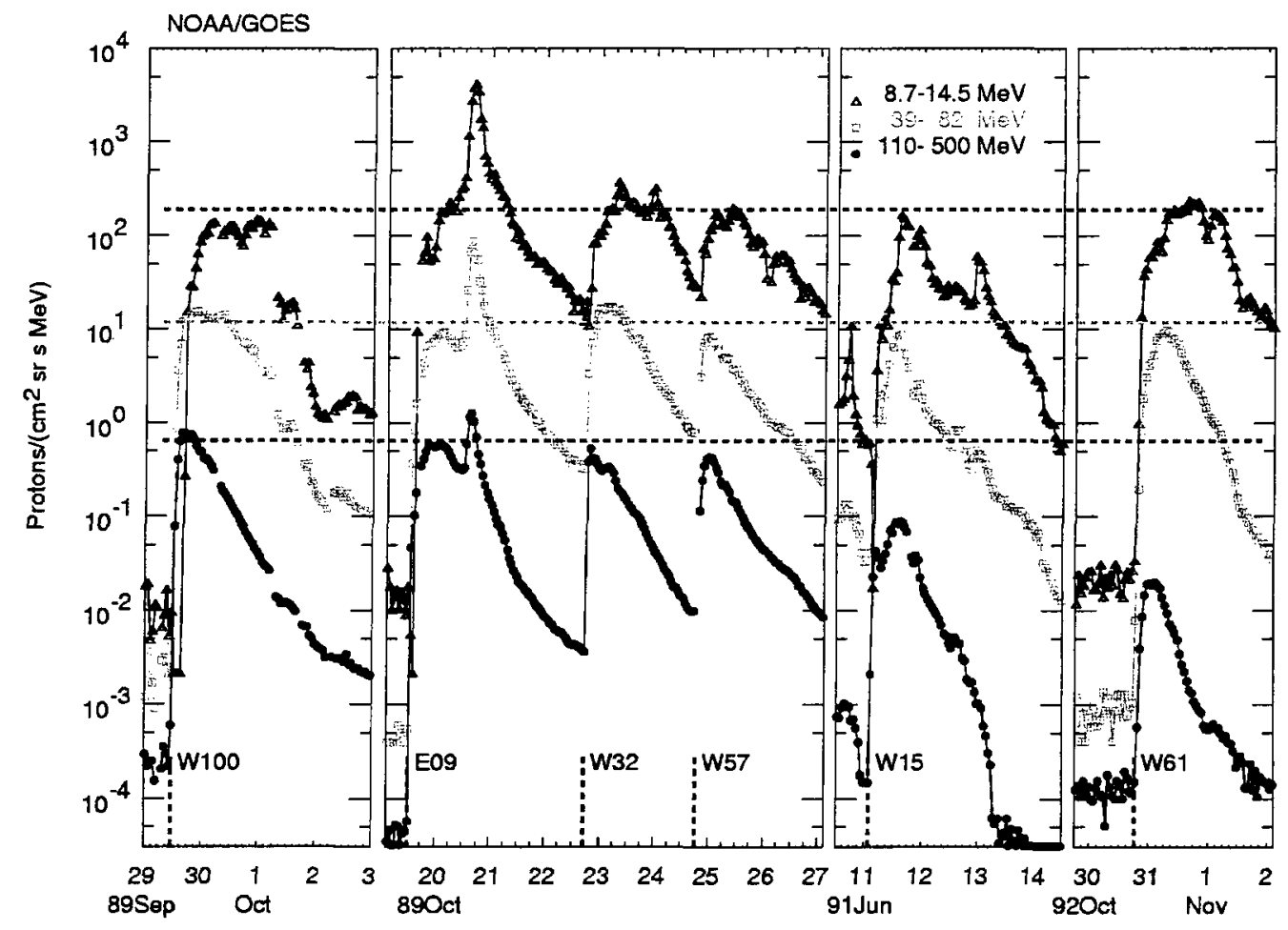

Fig. 7. Intensity-time profiles are shown for three energy intervals in six large events. Early in the events, the intensities do not rise above the respective streaming limits, indicated by the dashed lines. Only the shock peaks on 1989 October 20 exceed these limits (Reames and $\mathrm{Ng}$ 1998).

\section{ENERGY SPECTRA}

Energy spectra generated by a shock at equilibrium are power-law in form; the spectral power is related to the shock compression ratio (see Jones and Ellison 1991). The spectrum observed at a distance from the shock is then modified by transport as mentioned above. At high energies, however, the shock spectrum is modified because of decreased wave generation, the finite acceleration time, or spatial effects that cause high-energy particles to leak away from the shock. This leakage causes an exponential rollover of the spectra to produce a spectral form $E^{-\gamma} \exp \left(-E / E_{\text {knee }}\right)$ (see Ellison and Ramaty 1985).

The two spectra shown in Figure 8 have similar proton intensities below $\sim 10 \mathrm{MeV}$ but greatly different intensities at high energies. The location of the proton spectral "knee" is of great practical importance since $>100 \mathrm{MeV}$ protons penetrate spacecraft walls and astronaut's space suits, and plunge deeply into the Earth's polar atmosphere over a region that expands in size with increasing proton energy.

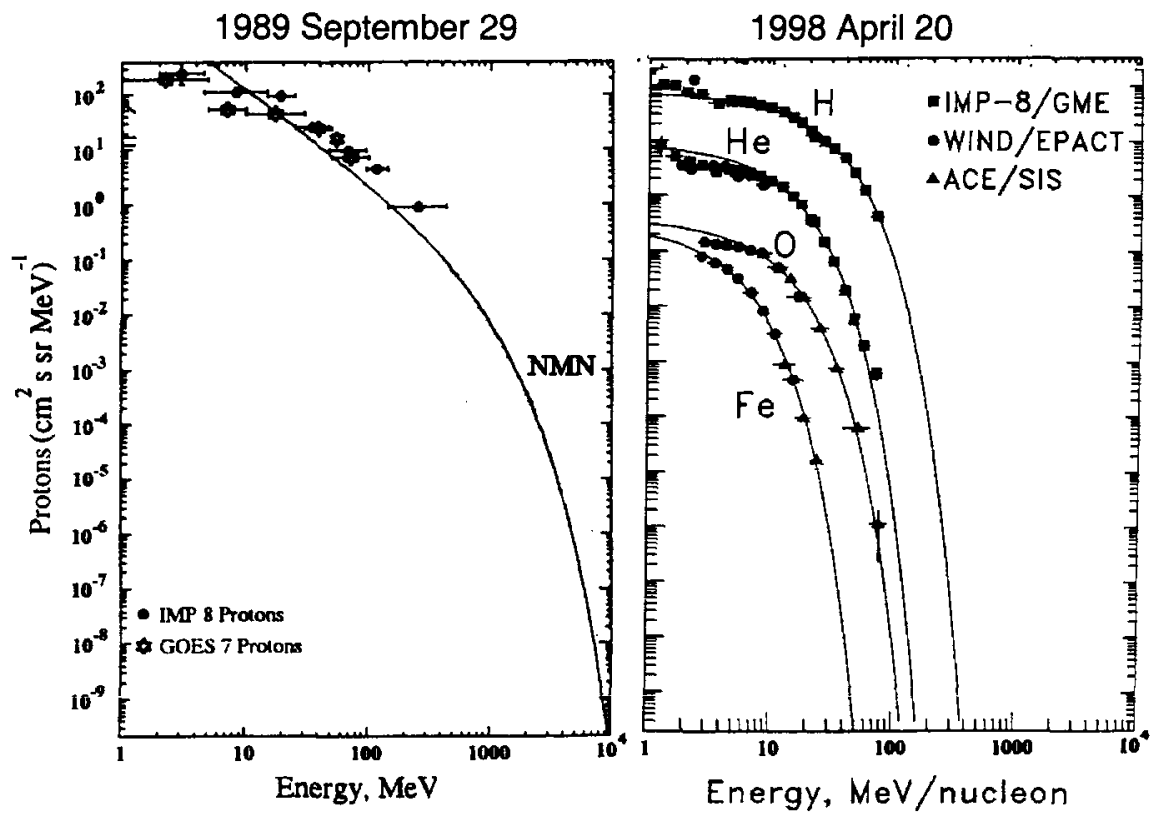

Fig. 8. Spectra in two events are fit to the Ellison and Ramaty (1985) form, $E^{-\gamma} \exp \left(-E / E_{\text {knee }}\right)$. In the 1989 September 29 event (Lovell et al. 1998) the knee energy of $\sim 1 \mathrm{GeV}$ falls in the region measured by the ground-level neutron monitor network (NMN). In the 1998 April 20 event (Tylka et al. 2000) the proton knee is at $15 \mathrm{MeV}$; for other species, $E_{\text {knee }}$ scales as $Q / A$ in this event. 


\section{YEARS OF SEP EVENTS}

The recent papers of McCracken et al. (2001a,b) summarize the behavior of SEP events during the last 400 years as deduced from impulsive increases in nitrates in polar ice cores. The nitrates are produced by the direct ionization of the upper polar atmosphere by SEP protons with energy nominally $>30 \mathrm{MeV}$ (Jackman et al. 1990, $2000,2001)$. Especially in the winter hemisphere, the nitrates are precipitated within 2-6 weeks in a snow layer that is eventually compressed into the polar firn and ice layers. The nitrate signal from the summer hemisphere is often reduced. Electrons precipitated during magnetic storms are also known to produce atmospheric nitrates but this evidently occurs at latitudes that are too low to affect the polar ice. The nitrate signal from SEP events is easily distinguished from that produced by volcanoes since the latter contains a large proportion of $\mathrm{H}_{2} \mathrm{SO}_{4}$ from volcanic $\mathrm{H}_{2} \mathrm{~S}$. However, known volcanoes are used to confirm the time base for the SEP measurements.

- Figure 9 shows a sample of the nitrate time record from McCracken et al. (2001a) that includes the time of the first white-light flare observed by Carrington (1860). This event has the largest fluence of the 400 - year record, $\sim 4$ times that of the 1972 August 4 event. Figure 10 compares the pattern of the large SEP events with that of the sunspot cycle.

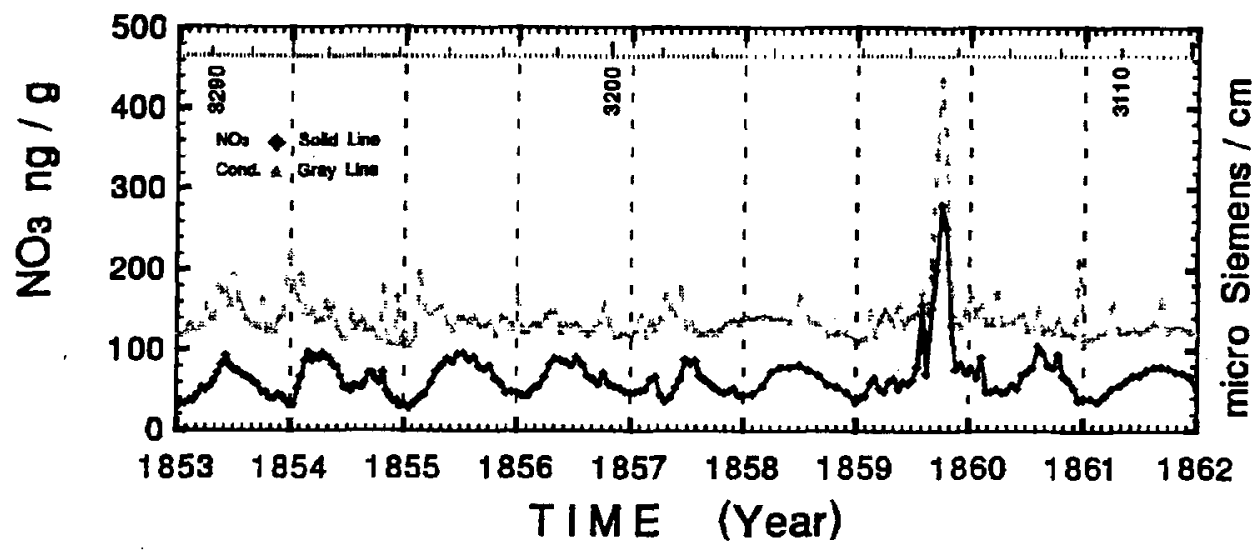

Fig. 9. An example of the $\mathrm{NO}_{3}$ and conductivity data showing the peak associated with the event observed by Carrington on 1859 September 1 (McCracken et al. 2001a).

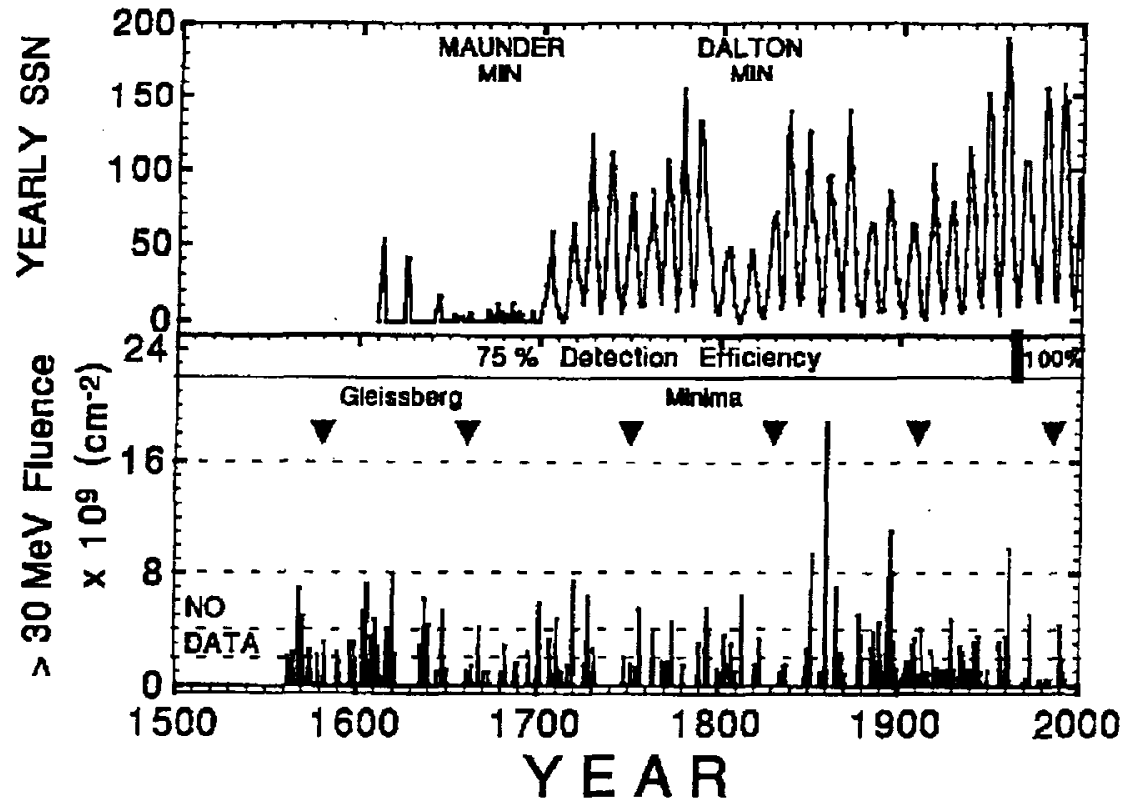

Fig. 10. Times of occurrence of SEP events with $>30 \mathrm{MeV}$ proton fluence exceeding $10^{9} \mathrm{~cm}^{-2}$ are shown in the lower panel. The pattern of the annual sunspot number is shown in the upper panel (McCracken et al. 2001b). 
In Figure 10 it is already clear that the temporal pattern of SEP events is not the same as that of sunspots. In fact, McCracken et al. (2001b) show that the SEP events follow the $\sim 80-100$ year Gleissberg cycle rather well. This is shown using the number of events per 11-year sunspot cycle in Figure 11.

Note that we are presently at a minimum in the Gleissberg cycle and we have been near this minimum for the entire time that instruments have been flown in space. Therefore, if the pattern continues, we can expect the next several solar cycles to have an increasing number of large SEP events, perhaps several times as many as we have seen in the last $\sim 30-40$ years.

\section{THE EVENT SIZE DISTRIBUTION}

The size distribution of SEP events, i.e. the probability of occurrence of a given intensity or of events that exceed a given fluence, is of great practical importance. It determines the risk of high radiation dose to humans and equipment in space and the probability of extensive ionization of the upper atmosphere.

Figure 12 shows distributions of intensity at three proton energy intervals measured by NOAA/GOES. Below the streaming limit at each energy interval, the distributions are well fit as a power-law, although there may be a slight excess immediately below the streaming limit. Above the streaming limit the distributions fall rapidly, times spent at these high-intensity values occur near the times of shock peaks, as noted in the left-hand panel. The probability of a strong shock peak depends upon occurrence of a CME, near central meridian on the Sun, that is fast and powerful enough

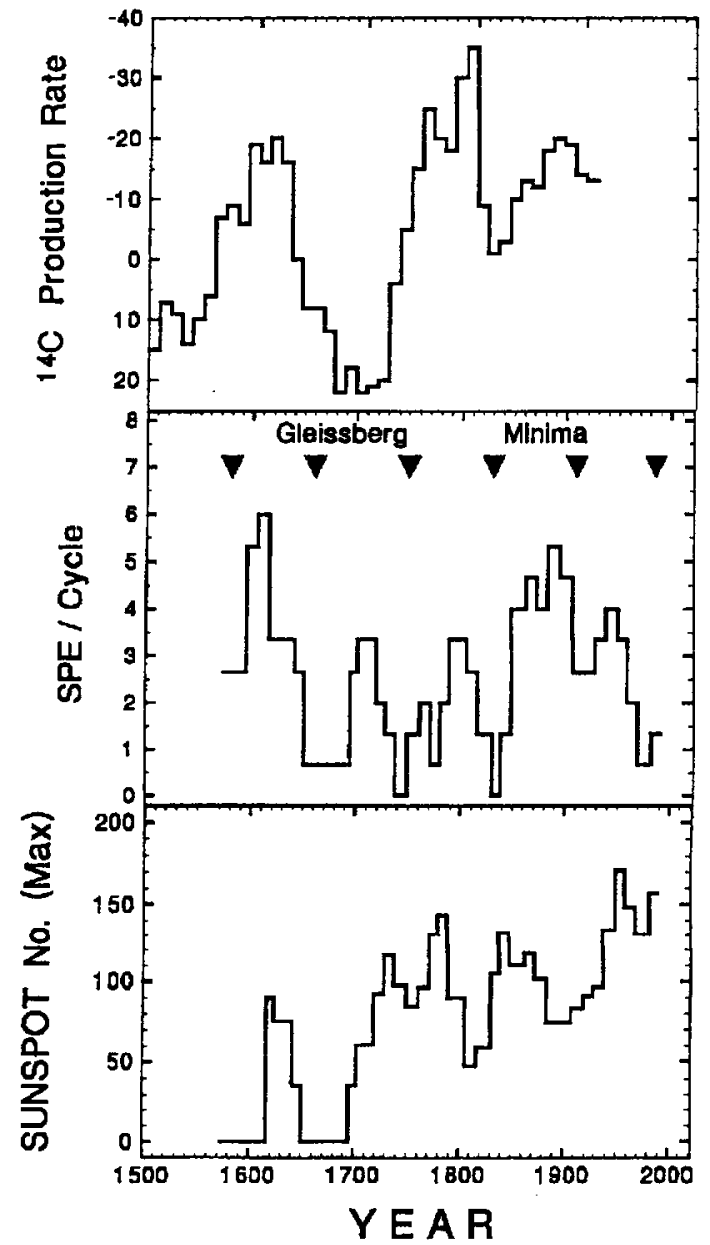

Fig. 11 Frequency of occurrence of SEP events is compared with that of sunspots and ${ }^{14} \mathrm{C}$ production (McCracken et al. 2001b).

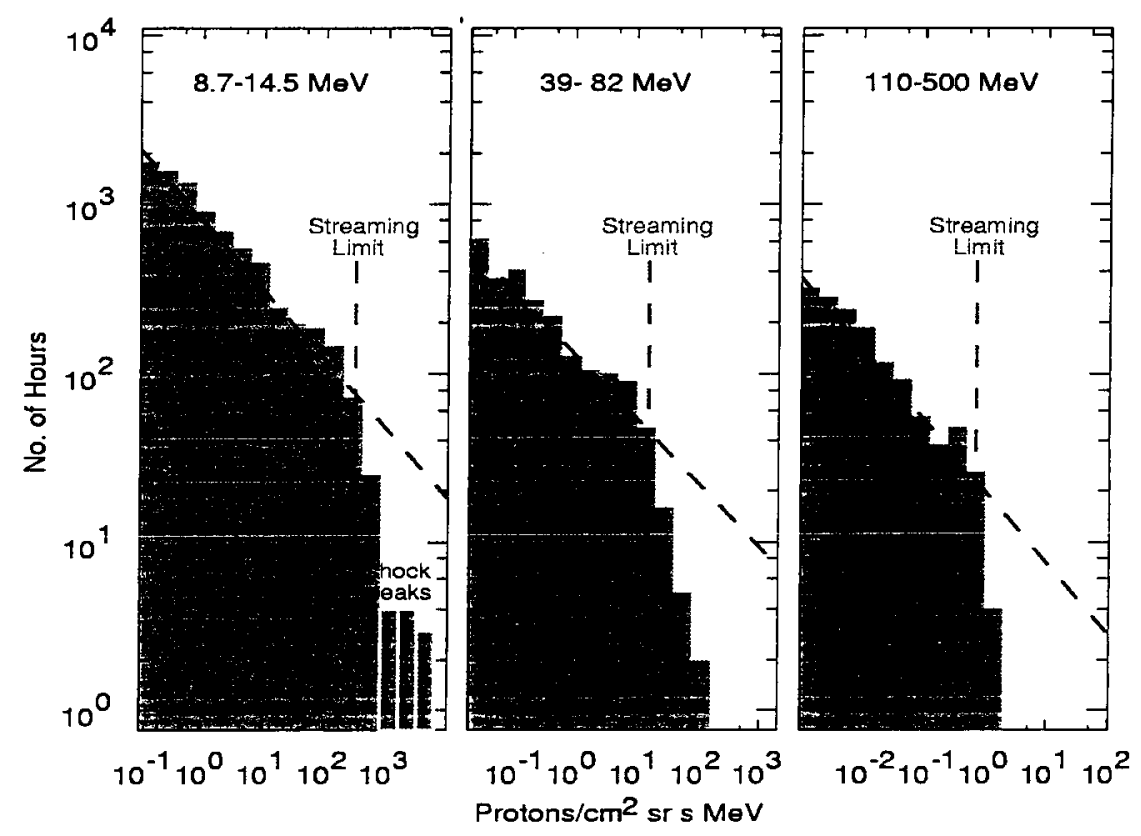

Fig. 12. The number of hours that the NOAA/GOES spacecraft spent at a given proton intensity during an -11-year period (January 1, 1986 to September 1, 1997) are shown for three different proton energy intervals. Hours with intensities above the streaming limit come near shock peaks, as noted on the figure. 
to drive a shock that remains sufficiently strong to continue accelerating high-energy particles even as it passes Earth. However, weaker CMEs from a wide band of solar longitude can drive shocks that accelerate particles near the Sun with intensities at the streaming limit. The latter are much more probable.

When we integrate over time and energy to determine the event fluence, well-defined physical boundaries such as the streaming limit become blurred by the varying time profiles of the events discussed above, and by presence of spectral knees. However, we may now cover a much longer time base that includes the nitrate observations (McCracken et al. 2001a,b) and the observation of radioactive secondaries produced in lunar rocks (Reedy 1996). The latter specify the average fluence over the lifetime of a specific radioactive nuclide but do not determine the number of events involved in that average.

Figure 13 shows the number of SEP events per year that exceed a given fluence as a function of fluence. Lines in the figure are determined, at low fluence, by the historic events and, at high fluence, by the upper limits derived from radioactive isotopes in lunar rocks (Reedy 1996). The large diamonds in the figure are obtained from the nitrate measurements (McCracken et al. 2001a). The latter are consistent with a rapid decrease in the rate of $>30 \mathrm{MeV}$ proton events at a fluence of $\sim 6 \times 10^{9} \mathrm{~cm}^{-2}$ that the authors as-

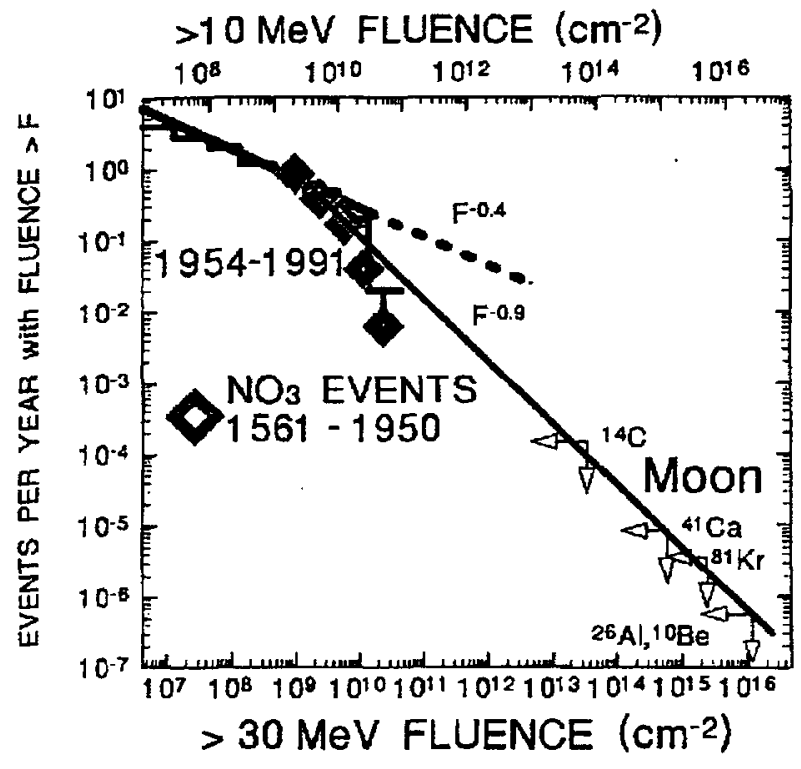

Fig. 13. The number of SEP events exceeding a given fluence is shown as a function of fluence (McCracken et al. 2001a). sociate with the streaming limit. It is likely that the streaming limit produces a rapid drop in the event rate, perhaps followed by new power-law dependence, rather than the mere change in slope that might be suggested by the lines in the figure.

\section{SUMMARY AND PROSPECTIVE}

Large SEP events have a significant effect on the Earth and on human enterprise. Protons $>30 \mathrm{MeV}$ penetrate space suits of astronauts and the spacecraft skin to produce a significant radiation hazard on the International Space Station and on missions to the moon or Mars. It is extremely difficult to shield protons $>100 \mathrm{MeV}$ without significant increases in spacecraft weight. SEP radiation at lower energies affects electronic circuits, solar cells and mirrored surfaces. SEP events ionize the polar mesosphere and stratosphere, affecting radio transmissions and profoundly altering the chemistry of the upper atmosphere. After the largest events, ozone levels can be affected for months and even years (e.g. Jackman et al. 2000).

The particles in large SEP events are accelerated at shock waves driven out from the sun by CMEs. The highest intensities of $<1 \mathrm{GeV}$ protons that are observed occur at the time of shock wave passage. Intensity-time profiles depend upon the size and speed of the CME and the location of the observer relative to it. Intense SEP peaks at shocks are associated with very fast CMEs near central meridian; otherwise, intensities are bounded at the streaming limit. Thus, the streaming limit produces a decrease in the probability of very large events.

How big can events be and how often do they occur? The answer to these questions is beginning to come from the nitrate record of the events in the polar ice. Improving the quality of measurements by obtaining ice cores from both poles and extending the record from 400 to 4000 years would greatly increase our knowledge of the event size distribution and of the stability of the solar cycles that control the event rate. The standard analysis of ice cores by glaciologists using 1- or 2-year time resolution is completely inadequate for SEP studies. The single most important measurement we can make to assess the SEP radiation risk to astronauts is to extend and improve the record of impulsive nitrate events in ice cores.

Unfortunately, however, ice core measurements do not tell us the particle spectrum, so the probability of large proton fluences at 100 or $1000 \mathrm{MeV}$ is uncertain. Protons in this energy range are highly penetrating and thus they are a serious radiation hazard that is difficult to shield. In the atmosphere, they interact to produce penetrating neutrons that irradiate passengers and flight crews in commercial aircraft flying polar routes. To address this problem we must understand the physics of energy spectral indices and spectral knees and the parameters that 
control this physics. Such studies are hampered by a lack of high-quality measurements of spectra and abundances above $\sim 100 \mathrm{MeV} / \mathrm{amu}$. Many instruments designed to measure high-energy galactic cosmic rays turn themselves off to avoid damage during intense SEP events.

Our ability to predict SEP events is almost nonexistent, but that does not prevent predictions from being made. In the author's opinion, reliable predictions of the onset and fluence of an SEP event prior to its occurrence are not likely in our lifetime. However, after an event onset, it should be possible to model the CME, the shock, and the acceleration and transport of particles sufficiently well to predict the peak intensity at shock passage and the event fluence. Such predictions are a tractable goal and could provide a useful warning of the highest SEP intensities many hours in advance.

\section{ACKNOWLEDGMENTS}

The author thanks M. A. Shea for her comments on the manuscript.

\section{REFERENCES}

Bell, A. R., The Acceleration of Cosmic Rays in Shock Fronts, Mon. Not. Roy. Astron. Soc., 182, 1471978.

Cane, H. V., D. V. Reames, and T. T. von Rosenvinge, The Role of Interplanetary Shocks in the Longitude Distribution of Solar Energetic Particles, J. Geophys. Res. 93, 9555, 1988.

Carrington, R. C., Description of a Singular Appearance Seen on the Sun on September 1, 1859, Mon. Not. Roy. Astron. Soc., 20, 13, 1860.

Ellison, D., and R. Ramaty, Shock Acceleration of Electrons and Ions in Solar Flares, Astrophys. J. 298, 400, 1985.

Gosling, J. T., The Solar Flare Myth, J. Geophys. Res., 98, 18949, 1993.

Jackman, C. H., A. R. Douglass, R. B. Rood, R. D. McPeters, and P. E. Meade, Effect of Solar Proton Events on the Middle Atmosphere during the Past Two Solar Cycles as Computed using a Two-dimensional Model, $J$. Geophys. Res., 95, 7417, 1990

Jackman, C. H., E. L. Fleming, and F. M. Vitt, Influence of Extremely Large Solar Proton Events in a Changing Stratosphere, J. Geophys. Res., 105, 11659, 2000.

Jackman, C. H., R. D. McPeters, G. J. Labow, and E. L. Fleming, Northern Hemisphere Atmospheric Effects due to the July 2000 Solar Proton Event, Geophys. Res. Lett., 28, 2883, 2001.

Jones, F. C., and D. E. Ellison, The Plasma Physics of Shock Acceleration, Space Sci. Revs. 58, 259, 1991.

Kahler, S. W., Solar Flares and Coronal Mass Ejections, Ann. Rev. Astron. Astrophys. 30, 113, 1992.

Kahler, S. W., Injection Profiles of Solar Energetic Particles as Functions of Coronal Mass Ejection Heights, Astrophys. J. 428, 837, 1994.

Kahler, S. W., The Correlation Between Energetic Particle Peak Intensities and Speeds of Coronal Mass Ejections: Effects of Ambient Particle Intensities and Energy Spectra, J. Geophys. Res. 106, 20947, 2001.

Kahler, S. W. and D. V. Reames, Probing the Magnetic Topologies of Magnetic Clouds by Means of Solar Energetic Particles, J. Geophys. Res. 96, 9419, 1991.

Kahler, S. W., N. R. Sheeley, Jr., R. A. Howard, M. J. Koomen, D. J. Michels, R. E. McGuire, T. T. von Rosenvinge, and D. V. Reames, Associations Between Coronal Mass Ejections and Solar Energetic Proton Events, J. Geophys. Res. 89, 9683, 1984.

Lee, M. A., Coupled Hydromagnetic Wave Excitation and Ion Acceleration at Interplanetary Traveling Shocks, $J$. Geophys. Res. 88, 6109, 1983.

Lee, M. A., Particle Acceleration and Transport at CME-Driven Shocks, in: Coronal Mass Ejections, edited by N. Crooker, J. A. Jocelyn, J. Feynman, Geophys. Monograph 99, (AGU press) 227, 1997.

Lovell, J. L., M. L. Duldig, J. E. Humble, An Extended Analysis of the September 1989 Cosmic Ray Ground Level Event, J. Geophys. Res. 103, 23,733, 1998.

McCracken, K. G., G. A. M. Dreschoff, E. J. Zeller, D. F. Smart, and M. A. Shea, Solar Cosmic Ray Events for the Period 1561-1994: 1. Identification in Polar Ice 1561-1950, J. Geophys. Res. 106, 21,585, 2001 a.

McCracken, K. G., G. A. M. Dreschoff, D. F. Smart, and M. A. Shea, Solar Cosmic Ray Events for the Period 1561-1994: 2. The Gleissberg Periodicity, J. Geophys. Res. 106, 21,599, $2001 \mathrm{~b}$.

Ng, C. K., and D. V. Reames, Focused Interplanetary Transport of $\sim 1 \mathrm{MeV}$ Solar Energetic Protons through SelfGenerated Alfven Waves, Astrophys. J. 424, 1032, 1994.

Ng, C. K., D. V. Reames, and A. J. Tylka, Effect of Proton-Amplified Waves on the Evolution of Solar Energetic Particle Composition in Gradual Events, Geophys. Res. Lett. 26, 2145, 1999. 
Reames, D. V., The Relationship Between Energetic Particles and Flare Properties for Impulsive Solar Flares, Astrophys. J. Suppl. 73, 235, 1990.

Reames, D. V., Solar Energetic Particles: A Paradigm Shift, Revs. Geophys. (Suppl.) 33, 585, 1995.

Reames, D. V., Particle Acceleration at the Sun and in the Heliosphere, Space Sci. Revs. 90, 413, 1999.

Reames, D. V., Magnetic Topology of Impulsive and Gradual Solar Energetic Particle Events, Astrophys. J. (Letters) 571, L63, 2002.

Reames, D. V., L. M. Barbier, and C. K. Ng, The Spatial Distribution of Particles Accelerated by CME-Driven Shocks, Astrophys. J. 466, 473, 1996.

Reames, D. V., S. W. Kahler, and C. K. Ng, Spatial and Temporal Invariance in the Spectra of Energetic Particles in Gradual Solar Events, Astrophys. J. 491, 414, 1997.

Reames, D. V. and C. K. Ng, Streaming-Limited Intensities of Solar Energetic Particles, Astrophys. J. 504, 1002, 1998.

Reames, D. V., and C. K. Ng, Angular Distributions of Fe/O from Wind: New Insights into SEP Transport, Astrophys. J. (Letters) 577, L59, 2002.

Reames, D. V., C. K. Ng, and Berdichevsky, D., Angular Distributions of Solar Energetic Particles, Astrophys. J. 550, 1064, 2001.

Reames, D. V., C. K. Ng, and A. J. Tylka, Initial Time Dependence of Abundances in Solar Particle Events, Astrophys. J. (Letters) 531, L83, 2000.

Reedy, R. C., Constraints on Solar Particle Events from Comparison of Recent Events and Million Year Averages, in Solar Drivers of Interplanetary and Terrestrial Disturbances, Vol. 95, ed. K. S. Balasubramanian, S. L Keil, and R. N. Smartt, (Astron Soc of the Pacific, San Francisco, CA) p 429, 1996.

Tylka, A. J., New Insights on Solar Energetic Particles from Wind and ACE, J. Geophys. Res. 106, 25333, 2001.

Tylka, A. J., P. R. Boberg, R. E. McGuire, C. K. Ng, and D. V. Reames, Temporal Evolution in the Spectra of Gradual Solar Energetic Particle Events, in Acceleration and Transport of Energetic Particles Observed in the Heliosphere, eds. R.A. Mewaldt, J.R. Jokipii, M.A. Lee, E. Moebius, and T.H. Zurbuchen, AIP Conf. Proc. 528, p 147, 2000.

Tylka, A. J., D. V. Reames, and C. K. Ng, Observations of Systematic Temporal Evolution in Elemental Composition during Gradual Solar Energetic Particle Events, Geophys. Res. Lett. 26, 2141, 1999. 